\title{
We André Bullinger (1941-2015)
}

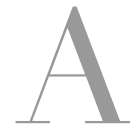

ndré Bullinger est mort mardi 3 février 2015 à Genève, là où il était né le 14 Mars 1941. Ce n'est pas seulement un savant de la psychologie développementale, connu internationalement pour ses recherches, que nous perdons, mais aussi un ami. Il aura grandement contribué à permettre une intégration rigoureuse des connaissances autour de la question du développement de l'enfant, notamment centré sur le stade sensori-moteur et ses avatars. Son dernier schéma, tel le tableau périodique des éléments de Mendeléiev ou la tablature pulsionnelle de Szondi/Schotte, résumant ses positions en la matière, restera un précieux apport et une féconde source de réflexions théoriques et pratiques pour tous ceux qui sont concernés par l'enfant, aussi bien les professionnels pédagogues, soignants, éducateurs et rééducateurs que les parents intéressés à un titre quelconque par les difficultés développementales et les handicaps de leur enfant. J'y reviendrai après avoir repris les quelques éléments de sa biographie universitaire que m'a transmis son épouse Anne Lise Bullinger.

Après des études techniques, il a entrepris des études de mathématiques. En parallèle, il occupait un emploi à temps partiel comme préparateur à l'Institut des Sciences de l'Éducation de l'Université de Genève. Par ce travail, il avait été amené à côtoyer Piaget, ce qui l'avait incité à commencer des études de psychologie. Après son diplôme et une licence en Sciences de l'Éducation (mention psychologie génétique), il a occupé pendant trois ans un poste de neuropsychologue à la clinique de neurologie de l'Hôpital Cantonal Universitaire de Genève. En 1968, Piaget lui offrit un poste d'assistant au laboratoire de psychologie expérimentale, couplé à un poste de collaborateur au Centre International d'Épistémologie Génétique. Ces emplois lui ont permis un contact proche avec les professeurs Inhelder, Tissot, de Ajuriaguerra et Piaget. Après une thèse de doctorat sous la direction de Jean Piaget portant sur les conduites de mesures chez l'enfant (1970), il a occupé le poste de chef de travaux du laboratoire de psychologie expérimentale (directeurs J. Piaget puis B. Inhelder).

Avec une bourse du Fond National Suisse de la Recherche Scientifique, il a ensuite effectué pendant trois ans des stages auprès de plusieurs groupes de recherche en Europe et aux États-Unis : chez les Professeurs E. Vurpillot (Paris), J. Bruner (Oxford), L.P. Lipsitt (Brown University), et M. Haith (Denver). De retour à Genève en 1976, il était nommé professeur assistant, puis professeur adjoint et, en 1989, professeur ordinaire de psychologie expérimentale à la Faculté de Psychologie et des Sciences de 
l'Éducation. Au cours de cette longue période, il a publié de très nombreux articles dans les revues internationales de psychologie, devenant progressivement une référence incontournable de la psychologie développementale. En 2002, il faisait valoir ses droits à la retraite de l'Université de Genève, et entreprenait de former de nombreuses équipes francophones au bilan sensori-moteur et à sa philosophie, notamment avec l'aide de Anja Kloeckner, de Dorotha Chadzinski et d'Évelyne Camaret dans les Universités de Lille, de Montpellier et de Brest. Il a participé personnellement à la formation de nombreuses équipes de CMP de secteurs et d'hôpitaux de jour de pédopsychiatrie (TED/TSA), de services de néonatalogie (soins de développement), de CAMSP (IMC, troubles du développements, troubles visuels) et d'établissements médico-éducatifs (troubles du développement et troubles cognitifs).

Le Fonds National Suisse de la Recherche Scientifique a subventionné la plus grande partie de ses travaux de recherche. Mais, non content de développer une théorie approfondissant notablement celles de ses prédécesseurs, Piaget, Inhelder, Ajuriaguerra et d'autres, il ressentit le besoin de mettre en pratique son expérience avec les enfants. Et en 1985, il a créé au sein de la Section de Psychologie, l'Atelier de l'Enfance, un service de consultation et de recherche centré sur les déficits sensoriels et moteurs du jeune enfant (malheureusement fermé de façon désinvolte par l'Université lors de sa retraite en 2002). De 1991 à 1994, il a assumé la charge de président de la Section de Psychologie au sein de l'Université de Genève. Il était consultant dans diverses institutions en Suisse Romande et en France, où ses interventions étaient très attendues par les praticiens engagés dans les soins et les rééducations des enfants en difficultés développementales.

Les thèmes de recherche qu'il a développés doivent beaucoup aux influences des personnes rencontrées. Si la perspective génétique et développementale offerte par Piaget joue un rôle essentiel, la dimension clinique offerte par les professeurs B. Inhelder, J. de Ajuriaguerra et, indirectement, par A. Rey, a permis d'enraciner son travail de recherche dans la psychologie de l'enfant. La rencontre avec Geneviève Haag sera également déterminante pour ce qui concerne ses travaux sur l'autisme infantile. À plusieurs reprises il a été invité comme professeur aux État-Unis, en France et en Belgique. Ces contacts lui ont permis, à travers des recherches menées en commun, de confronter son point de vue à d'autres perspectives théoriques. Son enseignement continue en France avec les trois personnes qu'il avait formées à cette fin : Anja Kloeckner, Dorotha Chadzinski et Évelyne Camaret.

On peut distinguer plusieurs périodes dans la production scientifique de Bullinger. Les premières recherches autonomes s'enracinent dans le courant de l'École de Genève et se développent dans une direction où les composantes techniques sont importantes et nouvelles pour l'époque. L'enregistrement de la motricité oculaire lui a permis de montrer, dans le développement, la nature instable du lien entre motricité oculaire et cognition. L'influence de la psychologie du travail, telle que l'a développée le professeur J. Leplat (Paris) et son équipe, a joué un rôle non 
négligeable dans cette prise en compte de la distance entre indices recueillis et activité psychique. Cette distinction entre fonctionnement sensorimoteur et activité est une des composantes de la perspective instrumentale que soutient Bullinger.

Dans une deuxième période, les recherches se sont orientées vers les déficits sensorimoteurs. L'exploration des déficits fonctionnels liés à la malvoyance ou à la cécité, ainsi que l'analyse du rôle de dispositifs de suppléances, tel le guide ultrasonique, ont permis de comprendre le lien étroit entre propriétés de la vision périphérique et régulation tonico-posturale chez le bébé et l'enfant. Partant de là, le champ des investigations cliniques s'est élargi à d'autres déficits, notamment à l'infirmité motrice cérébrale qui éclaire, sous un autre angle, la même problématique. L’appui sur le point de vue théorique de Wallon a orienté la réflexion théorique et l'approche clinique. La prise en compte du milieu humain, de l'émotion dans la régulation tonico-posturale a complété l'apport reconnu des flux sensoriels dans ce mécanisme. La perspective théorique qui apparaît dans une troisième période est appelée « instrumentale » et cherche à relier, sur le terrain de l'application clinique, les points de vue de Piaget et de Wallon.

Le début du développement est décrit comme une élaboration instrumentale des moyens sensorimoteurs : les flux sensoriels et les postures constituent les premiers matériaux de cette élaboration représentative de l'organisme. À travers la dimension tonique et posturale, dont la régulation dépend des flux sensoriels et de l'interaction avec le milieu humain, se forme un ensemble de représentations portant sur l'organisme et ses moyens sensorimoteurs, sur les objets et sur l'espace qui les contient. Dans cette constitution, l'interaction avec le milieu humain est essentielle, elle fait du bébé un petit d'homme. Pour pousser plus avant l'analyse de ces élaborations représentatives, l'observation des conduites des enfants autistes à divers moments de leur développement a permis de mieux comprendre le fonctionnement de l'enfant normal, obligeant à préciser les différents objets de connaissance que traitent ces enfants au cours de leur développement. Les derniers travaux concernent l'enfant prématuré. Ces recherches tentent de caractériser la spécificité de cette population et de son environnement. L’approche clinique comme outil de recherche fondamentale a permis de mettre à jour un ensemble de questions auxquelles la perspective instrumentale a donné des éléments de réponse. Les retombées de cette approche ne sont pas négligeables et offrent des voies intéressantes pour le diagnostic et la rééducation.

Plus largement, l'œuvre d'André Bullinger prend une place essentielle dans le travail psychologique développemental et psychopathologique de nombreux praticiens pour lesquels il a constitué un repère dans les nombreux troubles des enfants. Que ce soit auprès des bébés pour lesquels il a développé une approche à la fois très humaine et très rigoureuse de connaissance et de prise en compte de leurs difficultés propres, que ce soit auprès des enfants présentant ce qu'il avait lui-même appelé des " avatars du développement ", en faveur desquels il avait inventé les items de son bilan sensori-moteur, ses apports sont devenus désormais non seulement reconnus à leur 
juste valeur, mais en outre, suffisamment intériorisés pour constituer un socle solide pour leurs prises en charge complexes. Il a su analyser les difficultés des enfants présentant des infirmités motrices cérébrales, des enfants porteurs de handicaps visuels, des bébés prématurés avec des troubles de l'oralité, des enfants engagés dans un processus autistique, sans compter toutes les autres pathologies qu'il a pu côtoyer au cours de sa longue trajectoire professionnelle et pour lesquelles il avait toujours quelque avis à donner, en toute modestie, ce qui s'avérait très souvent d'une grande fécondité dans les réflexions des équipes qui les prenaient en charge.

En guise de témoignage de sa grande fécondité intellectuelle et humaine, je voudrais reprendre rapidement sa dernière synthèse du développement pour en montrer non seulement la pertinence évidente, mais surtout pour inciter les lecteurs intéressés à s'appuyer sur ses descriptions pour déployer de nouvelles pistes encore inexplorées. C'est sans doute ce qui lui plairait le plus : avoir permis de contribuer à ouvrir de nouvelles voies d'aide pour les enfants dans leur développement troublé. Pour les personnes intéressées par l'ensemble des problématiques envisagées, je ne peux que recommander la lecture des deux tomes de son Développement sensori-moteur de l'enfant et ses avatars. Un parcours de recherche, dont le premier est sorti en 2004, et dont le deuxième sortira en mai 2015.

André Bullinger inscrit le développement de l'enfant dans une continuité biologique qui va de la conception à l'âge adulte. Le premier chapitre (Soulé, Missonnier) anténatal de la vie de l'enfant est une période au cours de laquelle les éléments sensoriels résultant de la maturation progressive des organes sont pris en considération de façon rigoureuse pour construire les hypothèses d'un développement harmonieux, et en corollaire, celles d'un développement marqué par des avatars impactant à leur tour la trajectoire de l'enfant. Dans cette perspective, Bullinger passe en revue toutes les sensorialités pré-natales pour en écrire une histoire naturelle intégrant les acquis récents des sciences, et les mettre en articulation avec les sensorialités post-natales. Je ne reprendrai pas ici tous les détails du gigantesque travail qu'il a réalisé, mais quelques éléments qui peuvent en faire comprendre la richesse et la fécondité. En fidèle élève de Wallon, il tente de trouver l'origine d'un élément fondamental du développement interactif, celui de " dialogue tonique ». Avant même que cette expérience importante ait lieu dans le milieu aérien, il observe que le foetus dispose d'un réflexe d'extension postérieure en réponse à différentes irritations anté-natales, et que la paroi interne de l'utérus maternel constitue alors la réponse limitante de ce premier dialogue tonique intra-utérin. Cette expérience permet de comprendre l'importance des interventions humaines dès les premiers temps de vie aérienne pour assurer une continuité contenante aux réflexes du bébé de type hypertonie postérieure, déclenchés par différents éléments irritatifs, contenus ou non par les interprétations que la mère fait de ces premières expériences désagréables et par les solutions qu'elle proposera à son bébé pour en sortir. Bullinger conseillait de mettre le bébé en " petit paquet » pour lui permettre de retrouver des formes contenantes antérieures face aux angoisses 
désorganisantes. Un autre élément est venu prendre une place essentielle dans ses travaux plus récents, celui de la pesanteur. Le vécu de faible pesanteur par le foetus en anté-natal est soumis à la gravité universelle dès la naissance. Cela lui fait éprouver un vécu d'écrasement sensiblement différent, résultant de ses effets sur le corps du bébé, et contribue à déclencher les réflexes toniques qui aboutiront un an plus tard à la position debout. C'est “ l'espace de la pesanteur ». Là encore, Bullinger comprend une partie du développement de la première année du bébé comme la réponse à cette question physique fondamentale, et il propose de l'approcher par son concept de “ construction de la verticalité ». Mais la naissance n'est pas que le passage d'un milieu amniotique à un milieu aérien avec les conséquences que nous avons survolées. Elle comprend également la transition que le bébé effectue entre un nourrissage en continu par le sang maternel et son filtre placentaire en pré-natal, et un nourrissage fractionné qui va s'organiser dès lors autour de la bouche et de « l'espace oral ». Cet espace prendra une grande importance selon que le bébé peut conjoindre une fonction nutritive avec une autre fonction exploratrice des organes en question, constitués en espace complexe de connaissance du monde par l'oralité : bouche, langue, lèvres, joues, odorat, gustation, tonus sphinctérien de la bouche, déglutition. Sans oublier évidemment le fait que par ce même espace oral passe, lors des expirations du bébé, la colonne d'air qui donnera substance et forme aux pré-langage et langage. Mais Bullinger nous apprend que sans un appui à la fois sur le tonus, mais aussi sur le regard, périphérique puis fovéal, la subtile dialectique qui se déploie dans l'espace oral ne peut être engagée, et risque de devenir des impasses de différents ordres. Le tonus du dos et la qualité de son portage par l'adulte en charge de son développement constituent une base qui permet au bébé de regarder le monde qui lui est offert et d'en explorer les possibilités avec son espace oral, une fois les besoins nutritifs satisfaits. Sinon, il décrit les options pathologiques qui s'ouvrent devant lui, ce qui expliquera un certain nombre des symptômes retrouvés dans les pathologies archaïques : agrippements, stéréotypies. Une fois cet espace constitué de façon stable, le bébé doit construire “ l'espace du buste " pour tenir assis dans des conditions d'interactions favorables à son développement affectif et cognitif. Son tonus postérieur lui permet de se redresser sur sa colonne vertébrale (aspects biomécaniques développés par Roger Vasseur) et ainsi de diriger son regard vers un objet d'intérêt, et éventuellement d'accompagner déjà ce mouvement oculaire de celui de la main et plus précisément de l'index (fonction déictique des sémioticiens) lorsque l'instrumentation de la main le permettra, ainsi que de productions expiratoires accompagnant de sonorités ses émotions en rapport avec l'intérêt de la découverte ou des retrouvailles. Bullinger insiste en passant sur la fonction essentielle du " tonus pneumatique ", sorte de bouée tonique des poumons bloqués en inspiration, confortant le tonus postérieur, qui permet au bébé fragile ou encore immature de stabiliser son regard sur l'objet d'intérêt, mais alors, au détriment des productions sonores, à l'origine de dissociations entre l'exploration et le commentaire sonore des explorations. Une fois cet espace du buste stabilisé, l'enfant entreprend la construction de « l'espace 
du torse ». Là encore, l'observation fine effectuée par Bullinger lui permet de proposer un récit des fonctionnements autonomes de la main droite dans l'hémicorps droit et de la gauche dans l'hémicorps gauche, jusqu'à ce que chaque main, ambassadrice de son hémicorps, apporte à la bouche les objets d'intérêt dont elle complète grâce à ses fonctions exploratrices, la liste de leurs qualités sensorielles. La bouche devient dès lors le lieu des échanges entre droite et gauche. Les deux espaces droit et gauche sont alors mis en continuité par l'espace oral qui les rassemble pour constituer l'espace du torse, espace de rotation intégré dans lequel les mains exploratrices et le regard sont conjugués pour accroître les capacités de découverte du monde environnant. L'espace du torse corrélé à celui de la préhension manuelle et de l'exploration visuelle, en appui sur les réflexes ATNP, cöncide avec la maturation du tonus du bassin et des jambes, déverrouillant la position debout et ouvrant la période de découverte du bas du corps, bassin mobile et jambes porteuses, et ainsi celle de la marche et de conscientisation des fonctions sphinctériennes. Le corps devient un véhicule pour l'enfant, qui peut le mouvoir là où bon lui semble, développant chez lui l'impression de toute puissance infantile qui doit être bordée d'une nécessaire fonction limitante parentale. Chacune de ces étapes de l'espace du torse et du “ corps-véhicule " peut être l'occasion de clivages maintenant l'enfant dans des positions antérieures tels les clivages autistiques verticaux et horizontaux décrits par Geneviève Haag dans son étude clinique et psychopathologique des étapes de développement de l'enfant autiste infantile traité. Le clivage vertical maintient l'enfant avec deux hémicorps dissociés pouvant amener à de véritables hémiplégies autistiques, tandis que le clivage horizontal empêche l'accès à l'intégration du “ bas du corps », rendant la marche fluide et les fonctions de propreté inaccessibles chez certains enfants autistes. J'ai souligné ailleurs que la convergence entre André Bullinger et Geneviève Haag était pour moi le signe d'une rencontre féconde entre deux grands penseurs de la psychologie du vingtième siècle, puisqu'ils arrivaient aux mêmes constatations cliniques et théoriques par deux théories différentes devenant ainsi complémentaires. La description des deux ordres de clivages en est pour moi un indice majeur. Lors de nos nombreuses discussions, notamment dans le cadre de rencontres avec des " regards croisés "sur des situations cliniques (avec Louis Vallée, professeur de neuropédiatrie à Lille, Roger Vasseur, médecin de rééducation fonctionnelle au CAMSP de Villeneuve d'Ascq), nous avons souvent constaté que les scientifiques devraient davantage s'appuyer sur de telles expériences de convergences pour penser ensemble les observations cliniques, tant la complémentarité des approches semble aider à penser l'ensemble du développement des enfants et leurs avatars. André Bullinger incarne à mes yeux, une des personnes qui y a le plus contribué. Je ne saurai jamais assez le remercier de m'avoir fait l'amitié de travailler avec mes équipes successives et d'avoir contribué à ouvrir mes hypothèses de travail à de nombreuses reprises.

Pierre Delion 